\title{
Orbital stability of negative solitary waves
}

\author{
Nguyet Thanh Nguyen*, Henrik Kalisch \\ Department of Mathematics, University of Bergen, Johannes Brunsgate 12, 5008 Bergen, Norway
}

Available online 18 June 2009

\begin{abstract}
The generalized regularized long-wave equation admits a family of negative solitary waves. The stability of these waves is investigated by numerical simulation using a spectral discretization.
\end{abstract}

(C) 2009 IMACS. Published by Elsevier B.V. All rights reserved.

Keywords: Solitary waves; Orbital stability; Spectral discretization

\section{Introduction}

We will investigate the stability of the negative solitary-wave solutions of the generalized regularized long-wave equation

$$
u_{t}+u_{x}+\left(u^{p}\right)_{x}-u_{x x t}=0,
$$

where $p \geq 2$ is a positive integer. Note that for the values $p=2$ and $p=3$ in this equation, which is also called the generalized BBM equation, is used to model the propagation of small-amplitude surface waves on a fluid running in a long narrow channel $[6,14,17]$. Eq. (1.1) admits solitary-wave solutions of the form $u(x, t)=\Phi_{c}(x-c t)$. Indeed, when this ansatz is substituted into (1.1), there appears

$$
-c \Phi_{c}+\Phi_{c}+c \Phi_{c}^{\prime \prime}+\Phi_{c}^{p}=0
$$

and it is an elementary check that a particular solution of (1.2) is given by

$$
\Phi_{c}(x-c t)=A \operatorname{sech}^{\sigma}\left[K\left(x+x_{0}-c t\right)\right],
$$

where $\sigma=2 /(p-1), \quad K=(p-1) / 2 \sqrt{(c-1) / c}$, and

$$
A=\left[\frac{(p+1)(c-1)}{2}\right]^{1 /(p-1)} .
$$

These solutions are strictly positive progressive waves which propagate to the right without changing their profile over time. As can be seen from the expression (1.3), solitary waves with positive propagation velocity are defined only when $c>1$. For $p \leq 5$, these waves are always stable. However, if $p>5$ there exists a critical speed $c_{p}^{+}$such that the positive solitary waves are stable for $c>c_{p}^{+}$, and they are unstable for $1<c<c_{p}^{+}$. This result was proved by Souganidis and Strauss [16] using the general theory of Albert, Bona, Grillakis, Souganidis, Shatah and Strauss laid

\footnotetext{
* Corresponding author.

E-mail addresses: nguyet.nguyen@math.uib.no (N.T. Nguyen), henrik.kalisch@math.uib.no (H. Kalisch).
} 


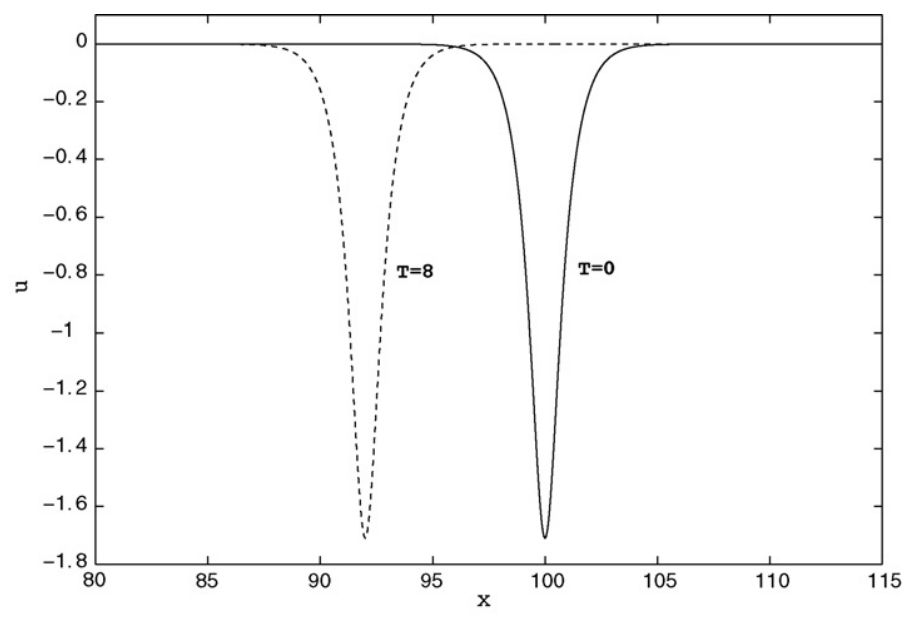

Fig. 1. Solitary wave of depression with speed $c=-1$. Here $p=4$.

down in [9,11], and pioneered by Boussinesq, Benjamin and coworkers $[4,7,10,15]$. For a thorough review of the results, and a numerical study of the stability of positive solitary waves, the reader may refer [8].

As also observed in [8], if $p \geq 2$ is odd, $-\Phi_{c}(x-c t)$ is also a solution of the generalized BBM equation. This follows immediately from the fact that $-u$ also satisfies Eq. (1.1). Thus, these are negative waves which also propagate to the right at the positive velocity $c>1$. Consequently, these negative solitary waves are always stable when $p \leq 5$. Otherwise, for $p>5$ these negative solitary waves are stable for speeds $c>c_{p}^{+}$, and they are unstable for $1<c<c_{p}^{+}$.

Finally, note that if $p \geq 2$ is even, then the formula (1.3) is valid for $c<0$ as well, resulting in negative solitary waves which propagate to the left. Surprisingly, these solitary waves can be unstable even if $p<5$. The main contribution of this paper is a numerical study of the stability of these negative solitary waves for both subcritical and supercritical $p$. Note that the case $p=2$ was already treated by one of the authors in [12].

A representative negative solitary wave is shown in Fig. 1, where $p=4$ and $c=-1$. In this calculation, the solution was approximated numerically from $T=0$ to $T=8$ and the size of the domain was 200. While Eq. (1.1) with quadratic and cubic nonlinearity is known to be a reasonable model for long waves of small-amplitude and negligible transverse variation, it is also apparent that the amplitudes of the negative solitary waves are of order 1 . Since the equation is given in dimensional variables, these solutions do not fall into the regime of physical validity of the equation as a long-wave model. In fact, this agrees with the observation explained in [5] that solitary waves of depression do not occur on the surface of fluids unless surface tension is very strong.

\section{Orbital stability}

As already observed by Benjamin and coworkers [4,6], a solitary wave cannot be stable in the strictest sense of the word. To understand this, consider two solitary waves of different height, centered initially at the same point. Since the two waves have different amplitudes they have different velocities according to the expression (1.3). As time passes the two waves will drift apart, no matter how small the initial difference was. However, in the situation just described, it is evident that two solitary waves with slightly differing height will stay similar in shape during the time evolution. Measuring the difference in shape therefore will give an acceptable notion of stability. This sense of orbital stability was introduced by Benjamin [4]. We say a solitary wave is orbitally stable if a solution $u$ of Eq. (1.1) that is initially sufficiently close to a solitary wave will always stay close to a translation of the solitary-wave during the time of evolution. A more mathematically precise definition is as follows. For any $\varepsilon>0$, consider the tube

$$
U_{\varepsilon}=\left\{u \in H^{1}: \inf _{s}\left\|u-\tau_{s} \Phi_{c}\right\|_{H^{1}}<\varepsilon\right\},
$$

where $\tau_{s} f(x)=f(x+s)$ is a translation of $f$. The set $U_{\varepsilon}$ is an $\varepsilon$-neighborhood of the collection of all translates of $\Phi_{c}$.

Definition 2.1. The solitary wave is stable if for all $\varepsilon>0$, there exists a $\delta>0$ such that if $u_{0}=u(\cdot, 0) \in U_{\delta}$, then $u(\cdot$, $t) \in U_{\varepsilon}$, for all $t \in \mathbb{R}$. The solitary wave $\Phi_{c}$ is unstable if $\Phi_{c}$ is not stable. 
The following two functionals $V$ and $E$ are critically important to the proof of stability of the solitary wave.

$$
\begin{aligned}
& V(g)=\frac{1}{2} \int_{-\infty}^{\infty}\left\{g^{2}+g_{x}^{2}\right\} d x, \\
& E(g)=\int_{-\infty}^{\infty}\left\{\frac{1}{2} g^{2}+\frac{1}{p+1} g^{p+1}\right\} d x .
\end{aligned}
$$

Observe that $V$ and $E$ are defined whenever $g \in H^{1}(\mathbb{R})$. In fact, note that $V(g)=\frac{1}{2}\|g\|_{H^{1}}^{2}$. Both $V(u)$ and $E(u)$ are conserved integrals, meaning that they are invariant in time if $u$ is a solution of the evolution equation (1.1). A proof of this fact can be found in [1]. Eq. (1.2) for the solitary waves can be written in terms of $V$ and $E$ as

$$
E^{\prime}\left(\Phi_{c}\right)-c V^{\prime}\left(\Phi_{c}\right)=0
$$

where $E^{\prime}$ and $V^{\prime}$ are the Fréchet derivatives of $E$ and $V$, respectively.

According to the general theory of Albert, Bona, Grillakis, Shatah, Souganidis, and Strauss in $[2,9,11,16]$, the stability of the solitary waves depends on the convexity of the function $d(c)=E\left(\Phi_{c}\right)-c V\left(\Phi_{c}\right)$. We have the following theorem:

Theorem 2.1. The solitary wave $\Phi_{c}$ is stable if the function $d$ is strictly convex, i.e, $d^{\prime \prime}(c)>0$. The solitary wave $\Phi_{c}$ is unstable if the function $d$ is strictly concave, i.e, $d^{\prime \prime}(c)<0$.

In the remainder of this section, we will find an explicit expression for the function $d(c)$. Note that calculations in this section are based on the results of Souganidis and Strauss [16], who consider a general class of evolution equations. The generalized BBM model presently in view is included as a particular case. However, Souganidis and Strauss only focus on the case of waves of elevation with positive velocity $c>0$. They find an expression for $d(c)$ of the form $\sqrt{c} F(c)$, for some function $F$. Since this form is obviously not valid in the case of negative $c$, we take a slightly different route of computing $d(c)$ explicitly. As was already noted in [12], the proof of Theorem 2.1 has to be modified somewhat to accommodate negative solitary waves. In the following, we use standard notation of mathematical analysis. In particular, we denote the standard inner product of two functions $f$ and $g$ in the real Hilbert space $L^{2}(\mathbb{R})$ by $\langle f, g\rangle$. The computation of $d(c)$ will be achieved by the following two lemmas.

Lemma 2.1. For $p \geq 2$ even and $c<0, d(c)=c\left\langle\Phi_{c}^{\prime \prime}, \Phi_{c}\right\rangle$.

Proof. For $\lambda>0$, let $\Phi_{c}^{\lambda}(\eta)=\Phi_{c}(\eta / \lambda)$. Then

$$
E\left(\Phi_{c}^{\lambda}\right)-c V\left(\Phi_{c}^{\lambda}\right)=\int_{-\infty}^{\infty}\left\{\lambda\left[\frac{1}{2} \Phi_{c}^{2}(z)+\frac{1}{p+1} \Phi_{c}^{p+1}(z)-\frac{c}{2} \Phi_{c}^{2}(z)\right]+c \frac{1}{2 \lambda} \Phi_{c}(z) \Phi_{c}^{\prime \prime}(z)\right\} d z .
$$

Next, we differentiate both sides of this equation with respect to $\lambda$, and evaluate at $\lambda=1$. From Eq. (2.1), it follows that

$$
\int_{-\infty}^{\infty}\left[\frac{1}{2} \Phi_{c}^{2}(z)+\frac{1}{p+1} \Phi_{c}^{p+1}(z)-\frac{c}{2} \Phi_{c}^{2}(z)\right] d z=\frac{c}{2} \int_{-\infty}^{\infty} \Phi_{c}(z) \Phi_{c}^{\prime \prime}(z) d z .
$$

Finally, using Eqs. (2.2) and (2.3), we have

$$
\begin{aligned}
d(c) & =\left[E\left(\Phi_{c}^{\lambda}\right)-c V\left(\Phi_{c}^{\lambda}\right)\right]_{\lambda=1} \\
& =\int_{-\infty}^{\infty}\left\{\left[\frac{1}{2} \Phi_{c}^{2}(z)+\frac{1}{p+1} \Phi_{c}^{p+1}(z)-\frac{c}{2} \Phi_{c}^{2}(z)\right]+\frac{c}{2} \Phi_{c}(z) \Phi_{c}^{\prime \prime}(z)\right\} d z \\
& =c \int_{-\infty}^{\infty} \Phi_{c}^{\prime \prime}(\eta) \Phi_{c}(\eta) d \eta .
\end{aligned}
$$


Lemma 2.2. For $p \geq 2$ even and $c<0$ the solution $\Phi_{c}$ of Eq. (1.2) can be written as

$$
\Phi_{c}(\eta)=(c-1)^{1 /(p-1)} \Psi\left(\sqrt{\frac{c-1}{c} \eta}\right),
$$

where $\Psi$ is the solution of the equation

$$
-\Psi^{\prime \prime}(\xi)+\Psi(\xi)-\Psi^{p}(\xi)=0 .
$$

The proof is a straightforward computation using the definition (2.4). In particular, note that $\Psi$ is independent of $c$.

Proposition 2.1. For $p \geq 2$ even and $c<0, d(c)=-b(c-1)^{\sigma+1} \sqrt{c /(c-1)}$, where $b=-\left\langle\Psi^{\prime \prime}, \Psi\right\rangle$, and $\sigma=2 /(p-$ 1).

Proof. Using Lemma 2.1, the definition of $\Psi$ in Eq. (2.4), and the change of variables $\xi=\sqrt{(c-1) / c} \eta$, it can be seen that

$$
\begin{aligned}
d(c) & =c \int_{-\infty}^{\infty} \Phi_{c}^{\prime \prime}(\eta) \Phi_{c}(\eta) d \eta \\
& =(c-1)^{(p+1) /(p-1)} \int_{-\infty}^{\infty} \Psi^{\prime \prime}\left(\sqrt{\frac{c-1}{c} \eta}\right) \Psi\left(\sqrt{\frac{c-1}{c} \eta}\right) d \eta \\
& =-b(c-1)^{\sigma+1} \sqrt{\frac{c}{c-1}}
\end{aligned}
$$

where $b=-\left\langle\Psi^{\prime \prime}, \Psi\right\rangle$, and $\sigma=(2 /(p-1))$.

Corollary 2.1. For $p \geq 2$ even and $c<0$, there is a negative critical speed

$$
c_{p}^{-}=\frac{1-\sqrt{2+\sigma^{-1}}}{2(\sigma+1)}, \text { where } \sigma=\frac{2}{p-1},
$$

such that the function $d(c)$ is strictly convex for $c<c_{p}^{-}$, and strictly concave for $c_{p}^{-}<c<0$.

Proof. From Lemma 2.1 it follows that $d(c)=-b(c-1)^{\sigma+1} \sqrt{c /(c-1)}$. Since $b$ is a constant independent of $c$, the first derivative of $d(c)$ is

$$
d^{\prime}(c)=-b\left[(\sigma+1)(c-1)^{\sigma} \sqrt{\frac{c}{c-1}}-\frac{1}{2}(c-1)^{\sigma-1} \sqrt{\frac{c-1}{c}}\right] .
$$

After some simplifications, it appears that the second derivative of $d(c)$ is

$$
d^{\prime \prime}(c)=-b \frac{(c-1)^{2 /(p-1)}}{c^{2}(c-1)} \sqrt{\frac{c}{c-1}} q(c),
$$

where $q(c) \equiv \sigma(\sigma+1) c^{2}-\sigma c-(1 / 4)$. We observe that $b$ is positive, and the second term in the expression is negative because $c<0$. Therefore, the sign of $d^{\prime \prime}(c)$ only depends on the sign of the quadratic polynomial $q(c)$. In particular, the sign of $d^{\prime \prime}(c)$ is the same as the sign of $q(c)$. Next, set $q(c)=0$, and solve this equation for $c$ (note that we are just interested in negative velocities $c<0)$. We find the critical speed $c_{p}^{-}$as

$$
c_{p}^{-}=\frac{\sigma-\sqrt{\sigma^{2}+\sigma^{2}+\sigma}}{2 \sigma(\sigma+1)}=\frac{1-\sqrt{2+\sigma^{-1}}}{2(\sigma+1)} .
$$




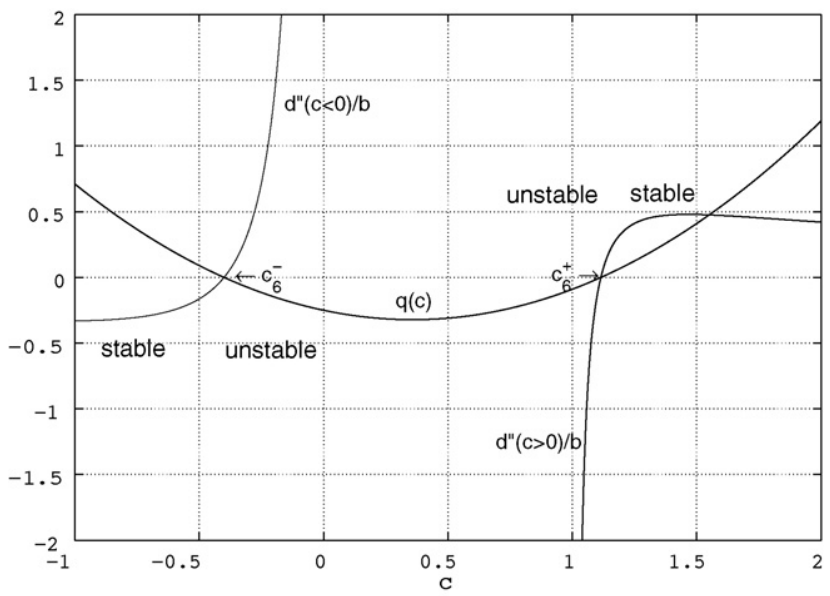

Fig. 2. $\left(d^{\prime \prime}(c)\right) / b$ and $q(c)$ for $p=6$, showing the stable and unstable regimes for both negative and positive speeds $c$. For $c<0$, the critical speed is $c_{6}^{-}=-0.4005$, and for $c>1$, the critical speed is $c_{6}^{+}=1.1148$.

Remark 2.1. Observe that the function $d(c)$ has different expressions for $c<0$ and $c>1$, respectively. ${ }^{1,2}$ However, the second derivatives share the quadratic polynomial $q(c)$ as a common factor. ${ }^{3}$ Moreover, the sign of $d^{\prime \prime}(c)$, for both $c<0$ and $c>1$ depends only on the sign of $q(c)$. Thus the negative and positive critical speeds are the negative and positive roots of $q(c)$, respectively. Fig. 2 illuminates this observation for the case $p=6$.

The following result is a consequence of Theorem 2.1 and Corollary 2.1 .

Corollary 2.2. Let $p \geq 2$ be an even integer, $\Phi_{c}$ be the solitary-wave solution (1.3) with negative velocity $c$, and $c_{p}^{-}$ be defined as in (2.5). The solitary wave $\Phi_{c}$ is stable if $c<c_{p}^{-}$, and unstable if $c_{p}^{-}<c<0$.

It should be mentioned that similar considerations possibly lead to the existence and stability or instability of negative solitary waves of other dispersive model equations, such as the generalized regularized Benjamin-Ono equation $[1,13]$ and the intermediate long-wave equation [3].

\section{Numerical simulation}

In the following, a numerical study is presented to illustrate the results obtained in the previous section. To discretize Eq. (1.1), we use a Fourier collocation method coupled with a four-stage Runge-Kutta time integration scheme.

For the purpose of numerical approximation, the problem is posed with periodic boundary conditions on the domain $x \in[0, L]$, where $L$ is sufficiently large. It was observed that $L=200$ was sufficient enough for the computations shown in this paper.

\subsection{Description of the numerical technique}

The problem (1.1) is translated to the interval $[0,2 \pi]$ by the scaling $u(a x, t)=v(x, t)$, where $a=(L / 2 \pi)$. The initial value problem is then

$$
\begin{cases}a^{2} v_{t}(x, t)+a v_{x}(x, t)+a\left(v^{p}\right)_{x}(x, t)-v_{x x t}(x, t)=0, & x \in[0,2 \pi], \quad t>0, \\ v(x, 0)=u_{0}(a x), & x \in[0,2 \pi], \quad t=0 \\ v(0, t)=v(2 \pi, t), & t \geq 0 .\end{cases}
$$

\footnotetext{
${ }^{1} d(c>1)=b c^{1 / 2}(c-1)^{\sigma+(1 / 2)}($ cf. [16]).

$2 d^{\prime}(c>1)=b\left[\frac{1}{2} c^{-(1 / 2)}(c-1)^{\sigma+(1 / 2)}+c^{(1 / 2)}\left(\sigma+\frac{1}{2}\right)(c-1)^{\sigma-(1 / 2)}\right]$.

${ }^{3} d^{\prime \prime}(c>1)=b c^{-(3 / 2)}(c-1)^{\sigma-(3 / 2)}\left[\sigma(\sigma+1) c^{2}-\sigma c-\frac{1}{4}\right]$ (cf. [16]).
} 
Table 1

Regularized long-wave equation; error due to temporal discretization. Here $p=4, T=8, L=200$, and $c=-1$.

\begin{tabular}{|c|c|c|c|c|c|c|}
\hline \multirow[t]{2}{*}{$h$} & \multicolumn{2}{|l|}{$N=2048$} & \multicolumn{2}{|l|}{$N=4096$} & \multicolumn{2}{|l|}{$N=6144$} \\
\hline & $L^{2}$-error & Ratio & $L^{2}$-error & Ratio & $L^{2}$-error & Ratio \\
\hline 0.1000 & 0.0031 & & 0.0031 & & 0.0031 & \\
\hline 0.0500 & $1.4002 \mathrm{e}-04$ & 22.140 & $1.4002 \mathrm{e}-04$ & 22.140 & $1.4002 \mathrm{e}-04$ & 22.140 \\
\hline 0.0250 & $6.8871 \mathrm{e}-06$ & 20.331 & $6.8871 \mathrm{e}-06$ & 20.331 & $6.8871 \mathrm{e}-06$ & 20.331 \\
\hline 0.0125 & $3.7087 \mathrm{e}-07$ & 18.570 & $3.7087 \mathrm{e}-07$ & 18.570 & $3.7087 \mathrm{e}-07$ & 18.570 \\
\hline 0.0063 & $2.2009 \mathrm{e}-08$ & 16.856 & $2.2009 \mathrm{e}-08$ & 16.856 & $2.2009 \mathrm{e}-08$ & 16.856 \\
\hline 0.0031 & $1.2318 \mathrm{e}-09$ & 17.867 & $1.2328 \mathrm{e}-09$ & 17.867 & $1.2328 \mathrm{e}-09$ & 17.867 \\
\hline 0.0016 & $9.8811 \mathrm{e}-11$ & 12.466 & $8.5453 e-11$ & 14.427 & $8.5446 \mathrm{e}-11$ & 14.428 \\
\hline 0.0008 & $4.9818 \mathrm{e}-11$ & 1.983 & $5.2716 \mathrm{e}-12$ & 16.210 & $5.2743 e-12$ & 16.200 \\
\hline 0.0004 & $4.9535 \mathrm{e}-11$ & 1.006 & $3.2082 \mathrm{e}-13$ & 16.432 & $3.1017 \mathrm{e}-13$ & 17.004 \\
\hline 0.0002 & $4.9534 \mathrm{e}-11$ & 1.000 & $1.8312 \mathrm{e}-14$ & 17.520 & $2.3061 \mathrm{e}-14$ & 13.450 \\
\hline 0.0001 & $4.9534 \mathrm{e}-11$ & 1.000 & $3.9972 \mathrm{e}-14$ & 0.458 & $2.6055 \mathrm{e}-14$ & 0.885 \\
\hline
\end{tabular}

The Fourier transform of the equation is

$$
\begin{cases}a^{2} \hat{v}_{t}+a i k \hat{v}+a i k \hat{v}^{p}+k^{2} \hat{v}_{t}=0, & t>0 \\ \hat{v}(k, t=0)=\mathscr{F}(v(x, 0), k), & t=0\end{cases}
$$

where $\mathscr{F}_{F}$ is the Fourier transform operator. Working in Fourier space, we can discretize the problem in the following forms:

$$
\begin{cases}\hat{v}_{t}(k, t)=-\frac{a i k}{a^{2}+k^{2}}\left[\hat{v}(k, t)+\mathscr{F}\left(\left[\mathscr{F}^{-1}(\hat{v})\right]^{p}, k\right)\right], & k=-\frac{N}{2}+1, \ldots, \frac{N}{2}, t>0, \\ \hat{v}_{t}(k, t)=0, & k=\frac{N}{2}, t>0, \\ \hat{v}(k, t=0)=\mathscr{F}(v(x, 0), k)=\frac{2 \pi}{N} \sum_{j=1}^{N} e^{-i k x_{j}} v\left(x_{j}, 0\right), & k=-\frac{N}{2}+1, \ldots, \frac{N}{2}-1, \quad t=0,\end{cases}
$$

where $\mathscr{F}^{-1}\left(\hat{v}, x_{j}\right)=\frac{1}{2 \pi} \sum_{k=-(N / 2)+1}^{N / 2} e^{i k x_{j}} \hat{v}(k, t)$ is the discrete inverse Fourier transform of $\hat{v}$ at the collocation points $x_{j}=(2 \pi j / N)$, for $j=1, \ldots, N$, where $N$ is an even number. This is a system of $N$ ordinary differential equations for the discrete Fourier coefficients $\hat{v}_{N}(k, t)$, for $k=-(N / 2)+1, \ldots,(N / 2)$. We integrate the system by using a four-stage explicit Runge-Kutta scheme with a uniform time step $h$.

To test the convergence of the algorithm and the numerical implementation, the normalized discrete $L^{2}$-norm is used. This norm is defined by

$$
\|v\|_{N, 2}^{2}=\frac{1}{N} \sum_{i=1}^{N}\left|v\left(x_{i}\right)\right|^{2} .
$$

The relative $L^{2}$-error is then defined to be

$$
\text { Error }=\frac{\left\|v-v_{N}\right\|_{N, 2}}{\|v\|_{N, 2}}
$$

where $v\left(x_{i}\right)$ is the exact solution, and $v_{N}\left(x_{i}\right)$ is the numerical approximate solution at a specific time $T$, for $i=1,2, \ldots, N$.

In the algorithm, the nonlinear term $v^{p}$ is treated pseudospectrally.

Consequently, there might be a significant danger of incurring aliasing errors, especially for high values of $p$. To minimize this source of error, a higher number of Fourier modes is used for larger values of $p$. To check the algorithm, we use the exact form (1.3) of the solitary wave as the initial value. Then, we calculate the relative $L^{2}$-error for various pairs of combinations between the time step $h=0.1 / 2 n$, for $n=1,2,3, \ldots$; and $N=2 m \times 1024$ for $m=1,2,3, \ldots$ The 
Table 2

Regularized long-wave equation; error due to temporal discretization. Here $p=6, T=2, L=200$, and $c=-2$.

\begin{tabular}{|c|c|c|c|c|c|c|}
\hline \multirow[t]{2}{*}{$h$} & \multicolumn{2}{|l|}{$N=4096$} & \multicolumn{2}{|l|}{$N=6144$} & \multicolumn{2}{|l|}{$N=8192$} \\
\hline & $L^{2}$-error & Ratio & $L^{2}$-error & Ratio & $L^{2}$-error & Ratio \\
\hline 0.1000 & 0.0567 & & 0.0567 & & 0.0567 & \\
\hline 0.0500 & 0.0030 & 19.0594 & 0.0030 & 19.0594 & 0.0030 & 19.0594 \\
\hline 0.0250 & $1.4661 \mathrm{e}-04$ & 20.4621 & $1.4661 \mathrm{e}-04$ & 20.4621 & $1.4661 \mathrm{e}-04$ & 20.4621 \\
\hline 0.0125 & $7.7498 \mathrm{e}-06$ & 18.9179 & $7.7498 \mathrm{e}-06$ & 18.9179 & $7.7498 \mathrm{e}-06$ & 18.9179 \\
\hline 0.0063 & $4.5168 \mathrm{e}-07$ & 17.1576 & $4.5168 \mathrm{e}-07$ & 17.1576 & $4.5168 \mathrm{e}-07$ & 17.1576 \\
\hline 0.0031 & $2.5049 \mathrm{e}-08$ & 18.0321 & $2.5049 \mathrm{e}-08$ & 18.0321 & $2.5049 \mathrm{e}-08$ & 18.0321 \\
\hline 0.0016 & $1.7286 \mathrm{e}-09$ & 14.4909 & $1.7286 \mathrm{e}-09$ & 14.4909 & $1.7286 \mathrm{e}-09$ & 14.4909 \\
\hline 0.0008 & $1.0638 \mathrm{e}-10$ & 16.2490 & $1.0637 \mathrm{e}-10$ & 16.2504 & $1.0638 \mathrm{e}-10$ & 16.2490 \\
\hline 0.0004 & $6.5867 \mathrm{e}-12$ & 16.1508 & $6.5867 \mathrm{e}-12$ & 16.1481 & $6.5939 \mathrm{e}-12$ & 16.1331 \\
\hline 0.0002 & $4.0666 \mathrm{e}-13$ & 16.1971 & $3.9242 \mathrm{e}-13$ & 16.7860 & $4.1137 \mathrm{e}-13$ & 16.0292 \\
\hline 0.0001 & $2.8866 \mathrm{e}-14$ & 14.0879 & $1.7318 \mathrm{e}-14$ & 22.6594 & $2.8147 \mathrm{e}-14$ & 14.6150 \\
\hline 0.00005 & $1.7372 \mathrm{e}-14$ & 1.6617 & $1.8587 \mathrm{e}-14$ & 0.9317 & $1.3269 \mathrm{e}-14$ & 2.1212 \\
\hline
\end{tabular}

Table 3

Regularized long-wave equation; error due to temporal discretization. Here $p=8, T=3, L=200$, and $c=-1.5$.

\begin{tabular}{|c|c|c|c|c|c|c|}
\hline \multirow[t]{2}{*}{$h$} & \multicolumn{2}{|l|}{$N=4096$} & \multicolumn{2}{|l|}{$N=6144$} & \multicolumn{2}{|l|}{$N=8192$} \\
\hline & $L^{2}$-error & Ratio & $L^{2}$-error & Ratio & $L^{2}$-error & Ratio \\
\hline 0.1 & 0.1534 & & 0.1534 & & 0.1534 & \\
\hline 0.05 & 0.0079 & 19.4279 & 0.0079 & 19.4279 & 0.0079 & 19.4279 \\
\hline 0.025 & $3.6317 \mathrm{e}-04$ & 21.7528 & $3.6317 \mathrm{e}-04$ & 21.7528 & $3.6317 \mathrm{e}-04$ & 21.7528 \\
\hline 0.0125 & $1.8163 \mathrm{e}-05$ & 19.9954 & $1.8163 \mathrm{e}-05$ & 19.9954 & $1.8163 \mathrm{e}-05$ & 19.9954 \\
\hline 0.0063 & $1.0210 \mathrm{e}-06$ & 17.7891 & $1.0210 \mathrm{e}-06$ & 17.7891 & $1.0210 \mathrm{e}-06$ & 17.7891 \\
\hline 0.0031 & $5.5285 \mathrm{e}-08$ & 18.4680 & $5.5285 \mathrm{e}-08$ & 18.4680 & $5.5285 \mathrm{e}-08$ & 18.4680 \\
\hline 0.0016 & $3.7670 \mathrm{e}-09$ & 14.6760 & $3.7669 \mathrm{e}-09$ & 14.6764 & $3.7670 \mathrm{e}-09$ & 14.6760 \\
\hline 0.0008 & $2.3171 \mathrm{e}-10$ & 16.2572 & $2.3023 e-10$ & 16.3616 & $2.3024 \mathrm{e}-10$ & 16.3611 \\
\hline 0.0004 & $2.9603 \mathrm{e}-11$ & 7.8273 & $1.4211 \mathrm{e}-11$ & 16.2003 & $1.4211 \mathrm{e}-11$ & 16.2012 \\
\hline 0.0002 & $2.5974 \mathrm{e}-11$ & 1.1397 & $8.6629 \mathrm{e}-13$ & 16.4044 & $8.9022 \mathrm{e}-13$ & 15.9634 \\
\hline 0.0001 & $2.5959 \mathrm{e}-11$ & 1.0006 & $3.4710 \mathrm{e}-14$ & 24.9580 & $4.8814 \mathrm{e}-14$ & 18.2371 \\
\hline 0.00005 & $2.5959 \mathrm{e}-11$ & 1.0000 & $1.4135 \mathrm{e}-14$ & 2.4555 & $2.7110 \mathrm{e}-14$ & 1.8006 \\
\hline 0.000025 & $2.5959 \mathrm{e}-11$ & 1.0000 & $1.7736 \mathrm{e}-14$ & 0.7970 & $2.5202 \mathrm{e}-14$ & 1.0757 \\
\hline
\end{tabular}

results are shown in Tables 1-3, where the solutions were approximated from $T=0$ to $T=8, T=2$, and $T=3$, with the speeds $c=-1, c=-2$, and $c=-1.5$, for values of $p$ as 4,6 and 8 , respectively.

The best combinations of spatial grid size $2 \pi / N$, and the time step $h$ to achieve the accuracy of to $10^{-14}$ for each value of $p$ are summarized in Table 4 .

For each value of $p$, a further increase in the number of Fourier modes, or a further reduction in the time step $h$ would not produce a smaller error. With an accuracy of $10^{-14}$, rounding errors probably play a role, so that it would not be prudent to increase $N$ or decrease $h$. While the numbers shown in Tables 1-3 focus mostly on the temporal convergence of the scheme, Fig. 3 shows the results of some runs aimed at validating the spatial convergence of the

Table 4

Most advantageous combinations of $N$ and $h$.

\begin{tabular}{llllll}
\hline$p=4$ & & $p=6$ & $p=8$ & \\
\hline$h$ & $N$ & $h$ & $N$ & $h$ & $N$ \\
\hline 0.0002 & 4096 & 0.0001 & 6144 & 0.00005 & 6144 \\
\hline
\end{tabular}





Fig. 3. Spectral accuracy of the spatial discretization for the regularized long-wave equation. Shown is the $L^{2}$-error as a function of the number of spatial gridpoints $N$. The time step is 0.0002 for $p=4,0.0001$ for $p=6$, and 0.00005 for $p=8$.

code. As expected, spectral convergence is achieved in these computations. From now on, all the results shown will be obtained by using the pairs of $N$ and $h$ as shown in Table 4 for each value of $p$.

\subsection{Experiments with stable negative solitary waves}

In order to study the stability of negative solitary waves, the exact formula (1.3) for $p=4,6,8$ and $c<c_{p}^{-}$is used. Initial data is chosen as a perturbation of the solitary wave in the amplitude. Thus, typical initial data has the following form

$$
u_{0}(x)=\gamma \Phi_{c}(x)
$$

where $\gamma$ represents the perturbation of the amplitude.

According to the theory in the previous section, the norm used to calculate the total error should be the $H^{1}$-norm. The discrete version of this norm is defined by,

$$
\|v(x)\|_{H^{1}, N}^{2}=\frac{1}{N} \sum_{i=1}^{N}\left(\left|v\left(x_{i}\right)\right|^{2}+\left|v_{x}\left(x_{i}\right)\right|^{2}\right) .
$$

The relative error in shape at a certain time $t$ is then defined by

$$
\frac{\inf _{s}\left\|\tau_{s} \Phi_{c}(a \cdot)-v_{N}(\cdot, t)\right\|_{H^{1}, N}}{\left\|\Phi_{c}(a \cdot)\right\|_{H^{1}, N}},
$$

where $u_{0}(x)=\gamma \Phi_{c}(x)$, and $v_{N}$ is the numerical approximation of the solution emanating from initial data being a perturbed solitary wave.

Fig. 4 displays the time evolution at several later times of a perturbed stable solitary wave with $c=-1.2$ in the case of $p=8$. The perturbations in the amplitude are $\gamma=0.975$ and $\gamma=1.025$. The figures show that the actual solutions propagate to the left, while their shape is still close to a translation of the solitary wave. Perturbing the amplitude of 



Fig. 4. Perturbation of stable solitary wave for $p=8$. On the left are results for $\gamma=0.975$. The graph on the lower left is a close-up of the dispersive tail. On the right are results for $\gamma=1.025$. The graph on the lower right is a close-up of the dispersive tail.

Table 5

The relative total error and conserved integrals at different times for a perturbed solitary wave. Here $L=200, N=4096$, and $h=0.0002$ are used.

\begin{tabular}{llrr}
\hline$p=4, \gamma=0.99, \bar{c}_{4}=-0.2612, c=-1$ & & & \\
\hline$t$ & Error in shape & & $E$ \\
\hline 0 & 0.0100 & 7.5972 & $-9.5165 \mathrm{e}-04$ \\
0.8 & 0.0242 & 7.6201 & $-9.5165 \mathrm{e}-04$ \\
1.6 & 0.0443 & 7.6640 & $-9.5165 \mathrm{e}-04$ \\
3.2 & 0.0452 & 7.7117 & $-9.5165 \mathrm{e}-04$ \\
6.4 & 0.0299 & 7.7175 & $-9.5165 \mathrm{e}-04$ \\
12.8 & 0.0162 & 7.7176 & $-9.5165 \mathrm{e}-04$ \\
25.6 & 0.0269 & 7.7176 & $-9.5165 \mathrm{e}-04$ \\
51.2 & 0.0205 & 7.7176 & $-9.5165 \mathrm{e}-04$ \\
\hline
\end{tabular}

the solitary waves positively or negatively appears to have an impact on the dispersive tail that remains after the bulk of the wave has moved off.

Tables 5 and 6 are displaying the relative total error of perturbed traveling waves, as well as the error in the conserved integrals $V$ and $E$. The tables show that for initial values differing by $1 \%$ (for $p=4$ ), and $0.8 \%$ (for $p=6$ and 8 ) from an exact solitary wave, the solutions have an error in shape of no more than $2-4.5 \%$ for $p=4,2-3.7 \%$ for $p=6$, and $2-5 \%$ for $p=8$. We also monitored discrete forms of conserved integrals $V$ and $E$, in these tables, and it appears that the conservation is excellent.

\subsection{Experiments with unstable negative solitary waves}

As in the experiments with stable negative solitary waves, initial data is chosen as an amplitude perturbation $\gamma$ of the solitary wave in the exact form (1.3), where $p=4,6$, and 8 are chosen for illustration, and the speeds $c$ are such that $c_{p}^{-}<c<0$. These computations showed that as time evolves, the solution is no longer close to a 
Table 6

The relative total errors and conserved integrals at different times for a perturbed solitary wave. Here $L=200, N=6144, h=0.0001$ for $p=6$, and $L=200, N=6144, h=0.00005$ for $p=8$.

\begin{tabular}{|c|c|c|c|c|c|c|c|}
\hline \multicolumn{4}{|c|}{$p=6, \gamma=0.992, \bar{c}_{6}=-0.4005, c=-2$} & \multicolumn{4}{|c|}{$p=8, \gamma=1.008, \bar{c}_{8}=-0.5231, c=-1.2$} \\
\hline$t$ & Error in shape & $V$ & $E$ & $t$ & Error in shape & $V$ & $E$ \\
\hline 0 & 0.0080 & 6.4007 & -0.0021 & 0 & 0.0080 & 5.7715 & $7.5181 \mathrm{e}-04$ \\
\hline 10 & 0.0375 & 6.4690 & -0.0021 & 10 & 0.0514 & 5.6434 & $7.5181 \mathrm{e}-04$ \\
\hline 20 & 0.0325 & 6.4690 & -0.0021 & 18 & 0.0424 & 5.6434 & $7.5181 \mathrm{e}-04$ \\
\hline 30 & 0.0264 & 6.4690 & -0.0021 & 28 & 0.0341 & 5.6434 & $7.5181 \mathrm{e}-04$ \\
\hline 40 & 0.0205 & 6.4690 & -0.0021 & 38 & 0.0267 & 5.6434 & $7.5181 \mathrm{e}-04$ \\
\hline
\end{tabular}

translation of the original solitary wave. Furthermore, resulting wave profiles are divided into two different cases. When $\gamma<1$, the wave disperses completely, developing into very strong oscillations over time. These results are shown in Fig. 5 for the case $p=4$. Experiments showed similar results for $p=6$ and 8. On the other hand, when $\gamma>1$, the solution approaches a larger solitary wave after leaving behind an oscillatory wavetrain. These results can be seen in Fig. 6. Observe that even though the same perturbation is used in all three cases in the computations shown in Fig. 6, the resulting solitary wave has a larger speed if $p$ is larger. This can be understood by considering the relation (1.4), which shows that for a given amplitude, the negative velocity is increasing (in absolute value) with increasing p.
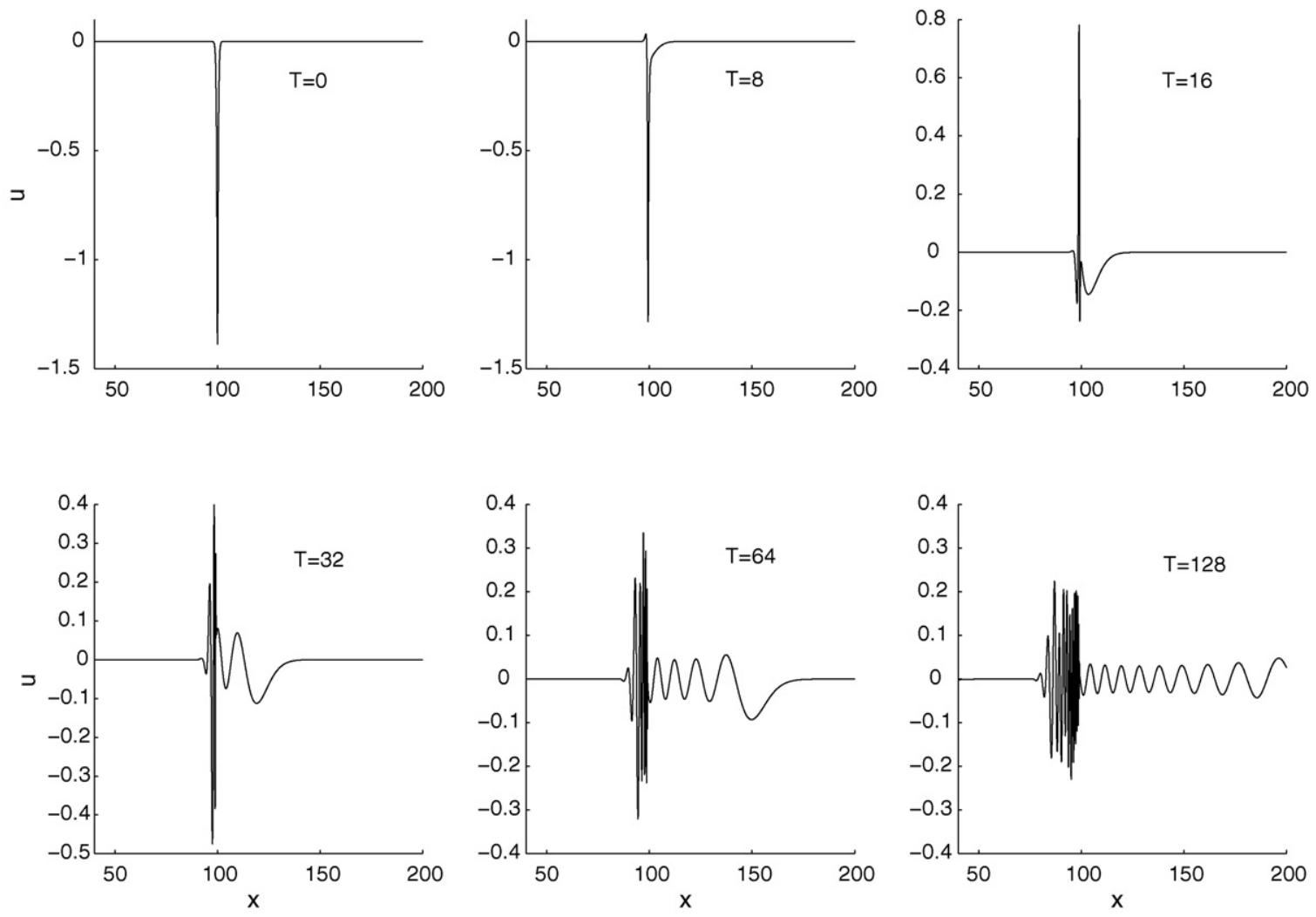

Fig. 5. Initial data: perturbed unstable solitary waves with $\gamma=0.99, c=-0.1$, and $p=4$. 

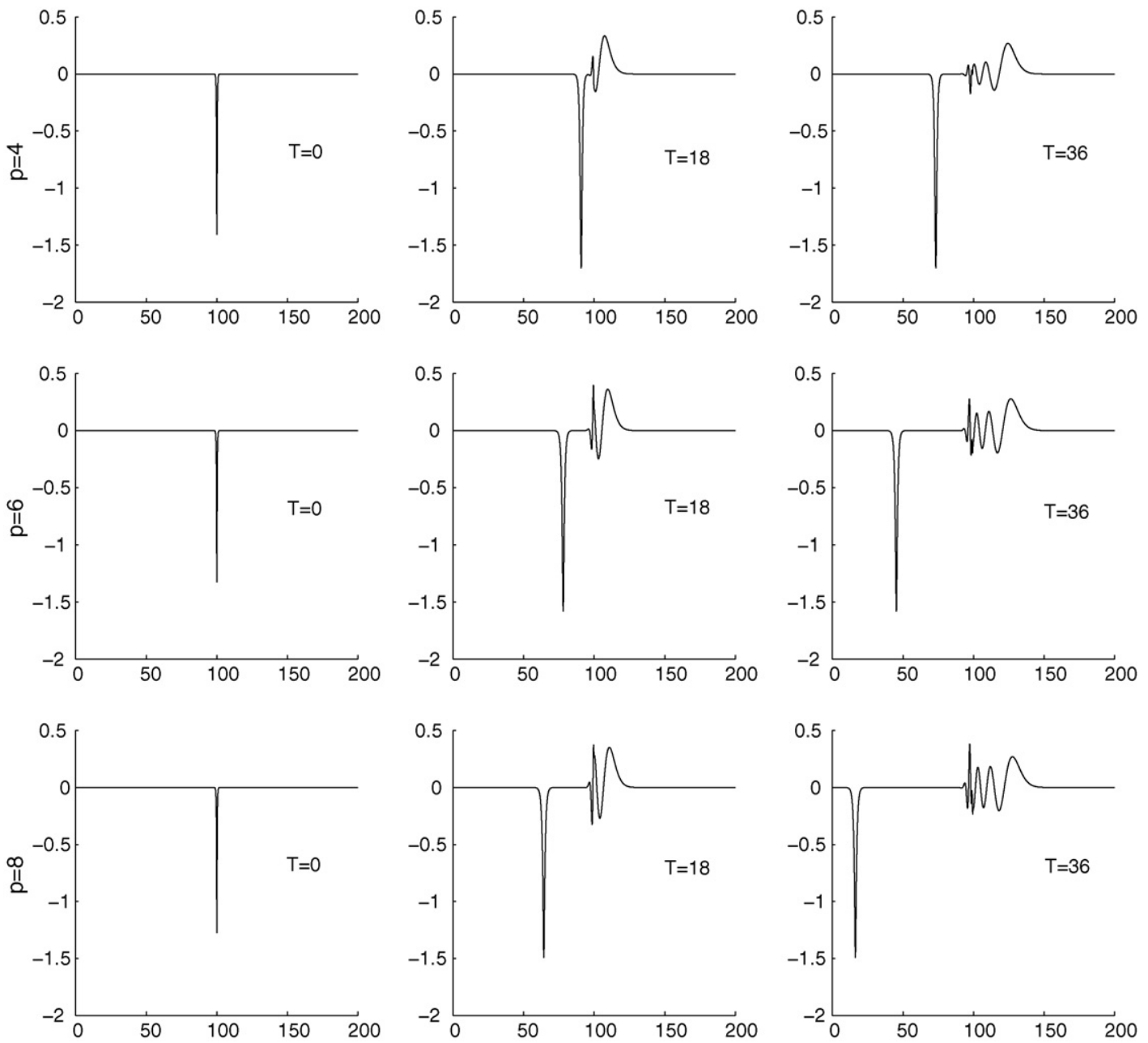

Fig. 6. Initial data: perturbed unstable solitary wave with $\gamma=1.02$, and $c=-0.05$.

\section{Acknowledgment}

This work was supported in part by the Research Council of Norway.

\section{References}

[1] J.P. Albert, J.L. Bona, Comparisons between model equations for long waves, J. Nonlinear Sci. 1 (1991) 345-374.

[2] J.P. Albert, J.L. Bona, Total positivity and the stability of internal waves in stratified fluids of finite depth, The Brooke Benjamin special issue (University Park, PA, 1989), IMA J. Appl. Math. 46 (1991) 1-19.

[3] J.P. Albert, J.F. Toland, On the exact solutions of the intermediate long-wave equation, Diff. Int. Eq. 7 (1994) 601-612.

[4] T.B. Benjamin, The stability of solitary waves, Proc. R. Soc. Lond. A 328 (1972) 153-183.

[5] T.B. Benjamin, The solitary wave with surface tension, Quart. Appl. Math. 40 (1982) 231-234.

[6] T.B. Benjamin, J.B. Bona, J.J. Mahony, Model equations for long waves in nonlinear dispersive systems, Philos. Trans. R. Soc. Lond. A 272 (1972) 47-78.

[7] J.L. Bona, On the stability theory of solitary waves, Proc. R. Soc. Lond. A 344 (1975) 363-374.

[8] J.L. Bona, W.R. McKinney, J.M. Restrepo, Stable and unstable solitary-wave solutions of the generalized regularized long-wave equation, J. Nonlinear Sci. 10 (2000) 603-638.

[9] J.L. Bona, P.E. Souganidis, W.A. Strauss, Stability and instability of solitary waves of Korteweg-de Vries type, Proc. R. Soc. Lond. A 411 (1987) 395-412.

[10] J. Boussinesq, Théorie des ondes et des remous qui se propagent le long d'un canal rectangulaire horizontal, en communiquant au liquide contenu dans ce canal des vitesses sensiblement pareilles de la surface au fond, J. Math. Pures Appl. 17 (1872) 55-108. 
[11] M. Grillakis, J. Shatah, W.A. Strauss, Stability theory of solitary waves in the presence of symmetry, J. Funct. Anal. 74 (1987) $160-197$.

[12] H. Kalisch, Solitary waves of depression, J. Comput. Anal. Appl. 8 (2006) 5-24.

[13] H. Kalisch, J.L. Bona, Models for internal waves in deep water, Disc. Cont. Dyn. Sys. 6 (2000) 1-19.

[14] P.G. Peregrine, Calculations of the development of an undular bore, J. Fluid Mech. 25 (1966) 321-330.

[15] J. Shatah, W. Strauss, Instability of nonlinear bound states, Commun. Math. Phys. 100 (1985) 173-190.

[16] P.E. Souganidis, W.A. Strauss, Instability of a class of dispersive solitary waves, Proc. R. Soc. Edinburgh 114A (1990) 195-212.

[17] G.B. Whitham, Linear and Nonlinear Waves, Wiley, New York, 1974. 